\title{
Variabilidade de parâmetros físico e químico na coluna de água em poços tubulares locados na zona de aflọramentos do Sistema Aquífero Guarani
}

\author{
Variability of physical and chemical parameters in the water column in wells in the \\ area outcrops aquifer Guarani system
}

\author{
Carlos Alberto Löbler ${ }^{1}$, Willian Fernando de Borba ${ }^{2}$, José Luiz Silvério da Silva ${ }^{3}$, \\ Iago Turba da Costa ${ }^{4}$ Lucas Lasta ${ }^{5}$
}

\begin{abstract}
${ }^{1}$ Geógrafo, Mestre em Engenharia Ambiental pela Universidade Federal de Santa Maria - UFSM, RS Brasil; carloslobler@gmail.com

${ }^{2}$ Engenheiro Ambiental e Sanitarista, Mestrando do Programa de Pós-graduação em Engenharia Ambiental da Universidade Federal de Santa Maria - UFSM, RS Brasil;

${ }^{3}$ Geólogo, Professor Doutor do Departamento de Geociências da Universidade Federal de Santa-UFSM, RS Brasil; ${ }^{4}$ Acadêmico do curso de Geografia Licenciatura da Universidade pela Universidade Federal de Santa Maria - UFSM, RS Brasil; ${ }^{5}$ Acadêmico do curso de Engenharia Sanitária e Ambiental da Universidade Federal de Santa Maria - UFSM, RS Brasil;
\end{abstract}

\begin{abstract}
Resumo
O Campus da Universidade Federal de Santa Maria (UFSM) é abastecido principalmente por águas subterrâneas, sendo essas captadas em Zona de Afloramentos (ZA) do Sistema Aquífero Guarani (SAG). O presente estudo tem por objetivo analisar duas variáveis, uma física que é a temperatura e uma química que é a Condutividade Elétrica (CE) na coluna da água em dois poços tubulares dedicados a pesquisa no Campus da UFSM. Estatisticamente, nas duas datas distintas de coletas, os dados apresentaram bastante uniformidade, sendo que a CE caracteriza pelo incremento do seu valor na medida em que se desce na coluna da água e a temperatura tente a decair à medida que se aprofunda na coluna da água. Houve algumas pequenas variações entre as diferentes datas de coleta para os mesmos poços, contudo constatou-se que prevaleceu a uniformidade dos dados na maioria dos casos.
\end{abstract}

Palavras-chave: Aquífero; Coluna de Água; Condutividade Elétrica; Temperatura.

\begin{abstract}
The campus of the Federal University of Santa Maria is mainly supplied by groundwater, and these captured in Outcrops Zone (ZA) of the Guarani Aquifer System (SAG). This study aims to examine two variables, a physics that is the temperature and a chemical which is the Electrical Conductivity (EC) in the water column in two wells dedicated to research on the campus of UFSM. Statistically, the two different dates of harvest, the data showed much uniformity, and the EC is characterized by the increase of its value in that it goes down in the water column and the temperature try to decay as it deepens in the water column. There were some minor variations between different times for the same collection wells, but it was found that the uniformity of data prevailed in most cases.
\end{abstract}

Keywords: Aquifer; Water Column; Electrical Conductivity; Temperature. 


\section{Introdução}

A água subterrânea é parte importante do ciclo hidrológico (Custodio e Silva Júnior 2008). Sendo que ela ocorre abaixo do nível de saturação ou nível freático, presentes nas formações geológicas aflorantes e parcialmente saturadas e nas formações geológicas profundas totalmente saturadas.

O abastecimento de água do Campus da Universidade Federal de Santa Maria (UFSM) é realizado principalmente por águas subterrâneas, as quais são captadas em Zona de Afloramentos (ZA) do Sistema Aquífero Guarani (SAG) (Silvério da Silva et al. 2012) em especial na unidade hidroestratigráfica Passo das tropas. No Campus ainda se encontram as unidades do Membro Alemão e Terrações Fluviais.

Segundo (Machado 2005) a constituição litológica do Aquífero Guarani no Rio Grande do Sul corresponde predominantemente de uma sucessão de camadas areníticas, intercaladas por outras mais argilosas plano-paralelas ou em forma de lente, e isto leva a uma dedução de que não se está em presença de um único aquífero, mas sim de um "sistema aquífero", onde suas camadas são intercalações de camadas com diferentes origens, ocasionando situações de diferentes porosidades/ permeabilidades com mais ou menos água disponíveis e que também irão influenciar em suas características físicas e químicas.

Diversos trabalhos testando diferentes metodologias têm sido utilizados em pesquisas de águas subterrâneas em poços tubulares ativos, e em poços dedicados no Campus da UFSM. Destacando-se os trabalhos coordenados ou apoiados pelo LABHIDROGEO do departamento de Geociências da UFSM. As captações de águas subterrâneas foram estudadas por Moreira (2005), Marion (2007 e 2009), D' Ávila (2009), Santiago (2010), Löbler et al. (2010, 2011, 2012, 2013), Silvério da Silva et al. (2012), Reckziegel (2012), Luiz (2013a, 2013b) Schuster (2013) e Löbler (2015). As pesquisas citadas de uma maneira geral apontaram o uso crescente das águas subterrâneas e ainda localmente com despejos de poluentes como os esgotos domésticos in natura.

O presente trabalho tem por objetivo analisar as variações de um parâmetro químico a Condutividade Elétrica (CE) e um parâmetro físico a temperatura, na coluna da água em dois poços tubulares desativados no Campus da UFSM, com espaço temporal de um ano. Para tanto se elaborou uma tabela estatística para auxiliar na interpretação dos dados coletados.

Entende-se por CE de uma água a capacidade que ela tem de conduzir eletricidade, estando diretamente relacionada à quantidade de sais dissolvidos sob a forma de íons (LIMA et al., 2014). A quantidade de sais dissolvidos na água subterrânea pode indicar inúmeros fatores, entre eles a perda de nutrientes do solo.

Silvério da Silva e Carmo (2013) e Carmo (2014) estudaram o comportamento da temperatura e da CE em ZA do SAG em uma microbacia do Rio Vacacaí-Mirim.

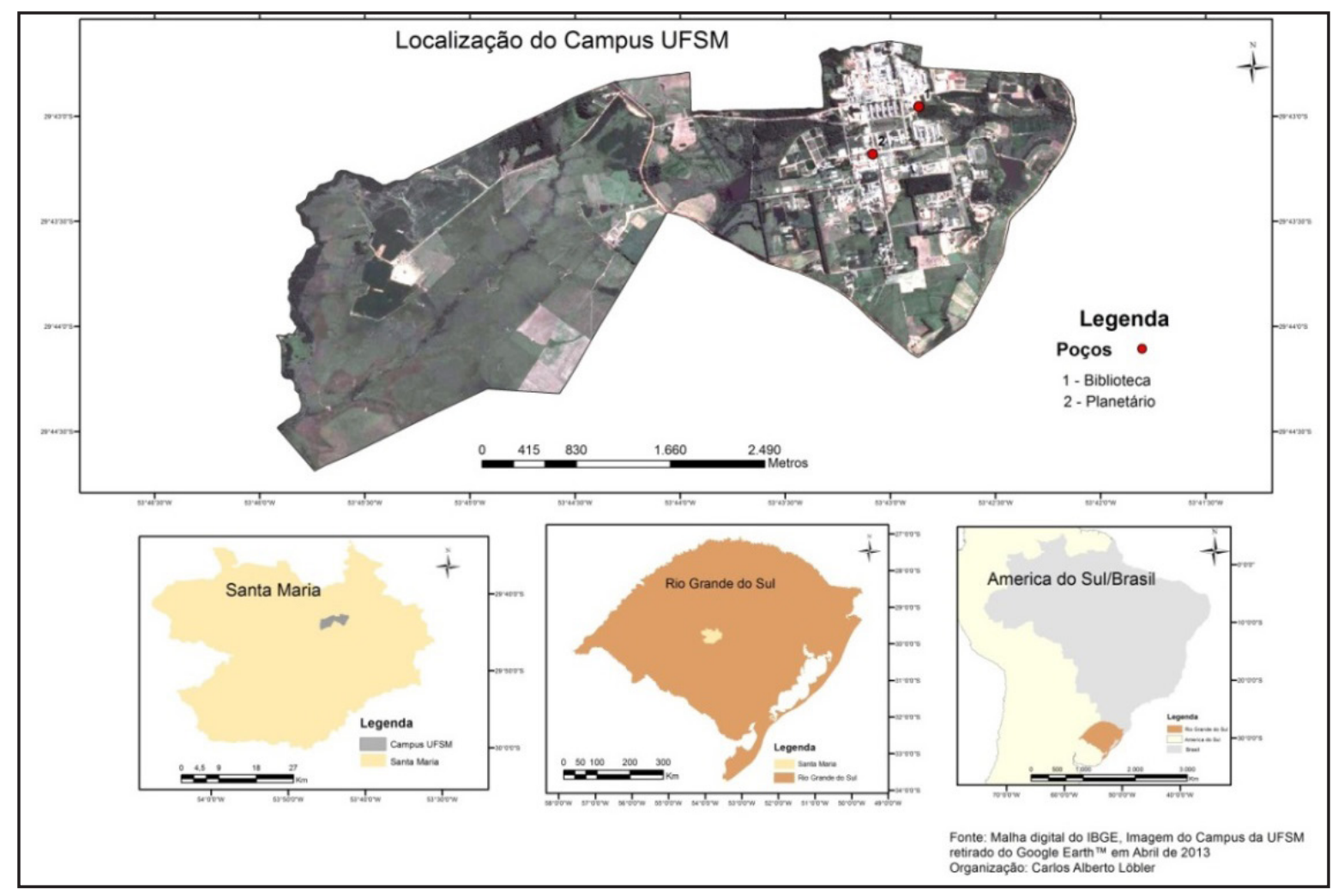

Figura 1- Mapa de localização da área de estudo com indicação dos poços dedicados 
Os autores encontraram variabilidade, principalmente no parâmetro CE nos poços rasos estudados.

\section{Caracterização da área de estudo}

O Campus da UFSM está localizado no bairro Camobi, na porção leste do município de Santa Maria, no estado do Rio Grande do Sul (RS), conforme é apresentado na figura 1. O Campus esta inserido em uma sub-bacia com rios afluentes do rio Vacacaí Mirim, fazendo parte da bacia hidrográfica dos rios Vacacaí e Vacacaí-Mirim (G60/SEMA/RS, 2005). Localizado na Depressão Central do estado na borda da Bacia Sedimentar do Paraná, em uma zona de transição entre os biomas Campos Sulinos (Pampa) e Mata Atlântica (VALENTINI, 2010). Ainda podem-se observar algumas espécies vegetais remanescentes dos dois biomas na área do Campus da UFSM.

Quanto à climatologia da área, conforme classificação de Köppen (KÖPPEN; GEIGER, 1928), o clima é subtropical úmido, tipo Cfa. Segundo Heldwein et al. (2009) as precipitações, através da base histórica de medidas entre os anos 1914-2009, registradas na estação meteorológica da UFSM, conveniada da rede INMET (Instituto Nacional de Meteorologia), apontam para médias anuais históricas de $1.712 \mathrm{~mm}$ e chuvas distribuídas para todos os meses do ano.

\section{Material e Método}

\section{Obtenção das informações}

Os dados utilizados para análise foram os registros de níveis da lâmina d'água, referentes à condutividade elétrica e à temperatura em cada metro da coluna de água até atingir o fundo da perfuração. As medições foram feitas em dois poços tubulares desativados do Campus: poço do Planetário e poço Biblioteca, os quais são poços dedicados a pesquisa, com profundidades 50 metros, (figura 2). Para realizar as medições usou-se o aparelho freatímetro sonoro TLC Solinst, de cabo de 100m (Figura 2).
Este aparelho é capaz de medir três diferentes parâmetros físicos: Condutividade Elétrica $\left(\mu \mathrm{S} . \mathrm{cm}^{-1}\right)$; Temperatura $\left({ }^{\circ} \mathrm{C}\right)$; e o Nível d'água $(\mathrm{m})$.

A metodologia utilizada para este estudo consiste na análise do comportamento de dois parâmetros, um físico que é a temperatura da água e um químico que é a $C E$, buscando-se através da análise estatística estabelecer um modelo hidrogeológico conceitual preliminar. Procura-se conhecer a dinâmica da água subterrânea inferindo o seu comportamento hidrodinâmico em duas datas distintas, ambas na estação do inverno (junho e setembro). As datas foram: primeira coleta no dia 16 de Julho de 2013 e a segunda coleta em 23 de agosto de 2014, portanto com espaço temporal de um ano entre os dois aferimentos. Executou-se com o auxílio do programa Microssoft Office Excel 2010 as analises estatísticas.

\section{Resultados e Discussão}

A partir dos trabalhos de campo realizados, em datas distintas nos anos de 2013 e 2014, nos dois poços, foi possível à construção da tabela 1 e os gráficos das figuras 3 , $4,5,6$. Nela apresenta-se a média dos dados avaliados, o desvio padrão, o máximo valor, o mínimo valor e a amplitude da variação (diferença entre o maior e o menor valor).

De maneira geral os dados de CE na primeira campanha de coleta dos dados (julho de 2013) apresentaram-se em média mais elevados (média de $1020 \mu$ S.cm-1 no poço Biblioteca e 509,3 MS.cm-1 no poço Planetário) em relação a segunda campanha (agosto de 2014 com média de 466 $\mu$ S.cm-1 para o poço biblioteca e $166 \mu$ S.cm-1 para o poço Planetário). Isso pode ser explicado por inúmeros fatores, entre eles o volume das chuvas ocorridas no período de aferimento nas datas, uma vez que um maior volume de recarga de água no aquífero provoca a diluição da água existente, tendendo a baixar a concentração de sais e consequentemente diminuir a CE.

Em relação à amplitude dos dados, que é a diferença entre o maior e o menor valor da amostragem, observase que os dados de CE e de temperatura nos dois poços

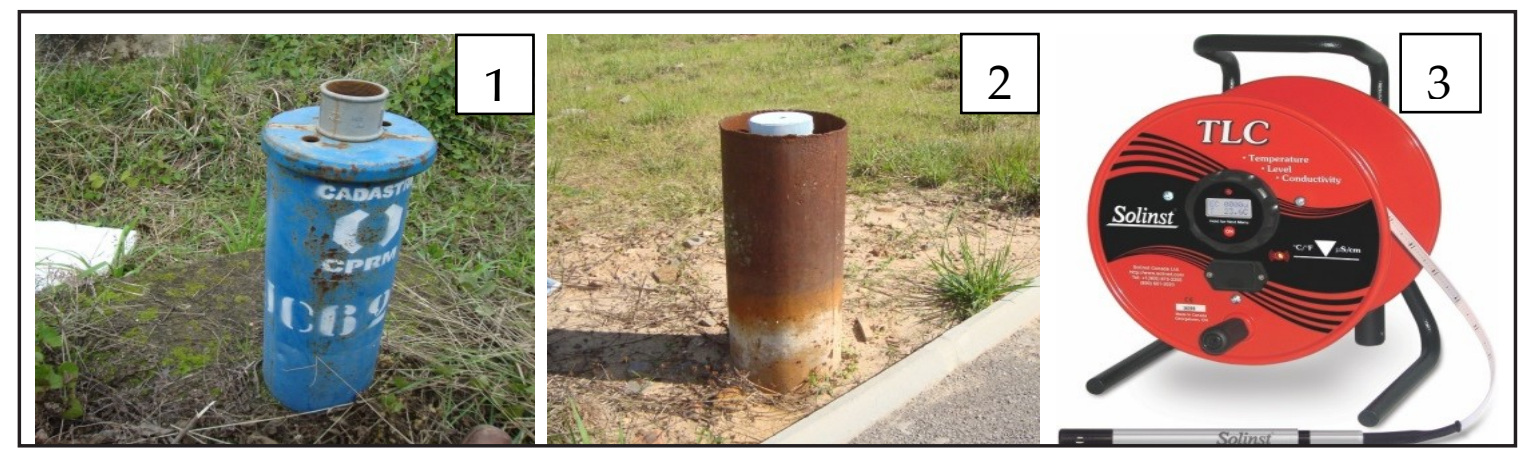

Figura 2 - 1 Poço Planetário, 2 Poço da Biblioteca e 3 aparelho freatímetro sonoro TLC Solinst usado nas medições dos parâmetros na coluna de água dos poços

Fonte: 1 e 2 Arquivo pessoal, 3 http://www.solinst.com/products/level-measurement-devices/107-tlc-meter/ 
Tabela 1 - Dados medidos na coluna de água nos poços: Planetário e Biblioteca no Campus da UFSM no dia 16 de Junho de 2013 e 23 de Agosto de 2014

\begin{tabular}{|c|c|c|c|c|c|c|c|c|}
\hline Poços & \multicolumn{4}{|c|}{ Poço Biblioteca } & \multicolumn{4}{|c|}{ Poço Planetário } \\
\hline Data & \multicolumn{2}{|c|}{ 16/07/2013 } & \multicolumn{2}{|c|}{$23 / 08 / 2014$} & \multicolumn{2}{|c|}{ 16/07/2013 } & \multicolumn{2}{|c|}{$23 / 08 / 2014$} \\
\hline Parâmetros & ${ }^{*} \mathrm{CE}$ & ${ }^{* *} \mathrm{~T}\left({ }^{\circ} \mathrm{C}\right)$ & $\mathrm{CE}$ & $\mathrm{T}\left({ }^{\circ} \mathrm{C}\right)$ & $\mathrm{CE}$ & $\mathrm{T}\left({ }^{\circ} \mathrm{C}\right)$ & $\mathrm{CE}$ & $\mathrm{T}\left({ }^{\circ} \mathrm{C}\right)$ \\
\hline Média & 1020,2 & 24,7 & 466,0 & 23,1 & 509,3 & 25,7 & 160,4 & 23,9 \\
\hline Desvio Padrão & 67,0 & 0,18 & 47,6 & 0,4 & 152,0 & 0,18 & 53,99 & 0,27 \\
\hline Máximo & 1087,0 & 25,0 & 533,0 & 24,0 & 601,0 & 25,9 & 259,0 & 24,5 \\
\hline Mínimo & 799,0 & 24,5 & 298,0 & 22,7 & 136,0 & 25,4 & 78,0 & 23,60 \\
\hline Amplitude & 288,0 & 0,5 & 235,0 & 1,3 & 465,0 & 0,5 & 181,0 & 0,9 \\
\hline $\mathrm{R}^{2}$ & 0,53 & 0,90 & 0,71 & 0,86 & 0,31 & 0,93 & 0,83 & 0,96 \\
\hline $\begin{array}{l}\text { Correlação com o } \\
\text { nível }\end{array}$ & 0,730 & $-0,948$ & 0,843 & $-0,930$ & 0,555 & $-0,966$ & 0,910 & $-0,980$ \\
\hline Correlação CE e T & \multicolumn{2}{|l|}{$-0,709$} & \multicolumn{2}{|l|}{$-0,896$} & \multicolumn{2}{|l|}{$-0,440$} & \multicolumn{2}{|l|}{$-0,905$} \\
\hline
\end{tabular}

* Condutividade Elétrica $\left(\mu \mathrm{S} . \mathrm{cm}^{-1}\right.$ ** Temperatura $\left({ }^{\circ} \mathrm{C}\right)$

Fonte: Trabalhos de campo realizados em junho de 2013 e agosto de 2014

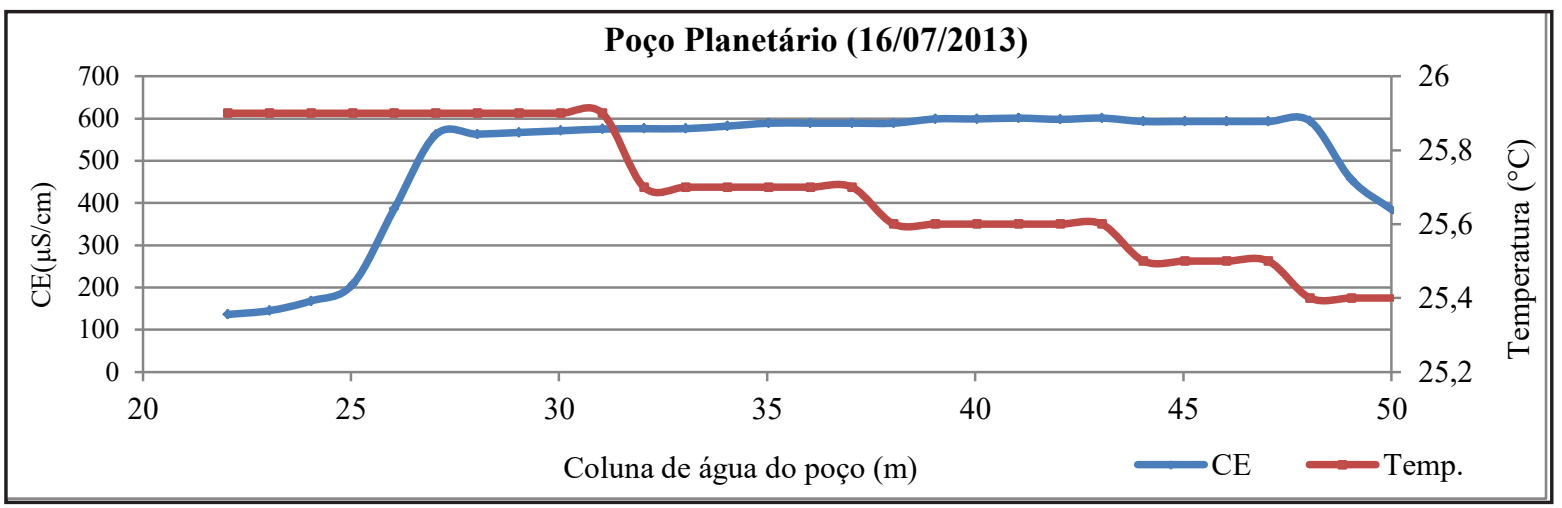

Figura 3 - Variação de CE e temperatura da água ao longo da coluna da água no poço Planetário no dia 16/07/2013 Fonte: Trabalho de campo, julho de 2013

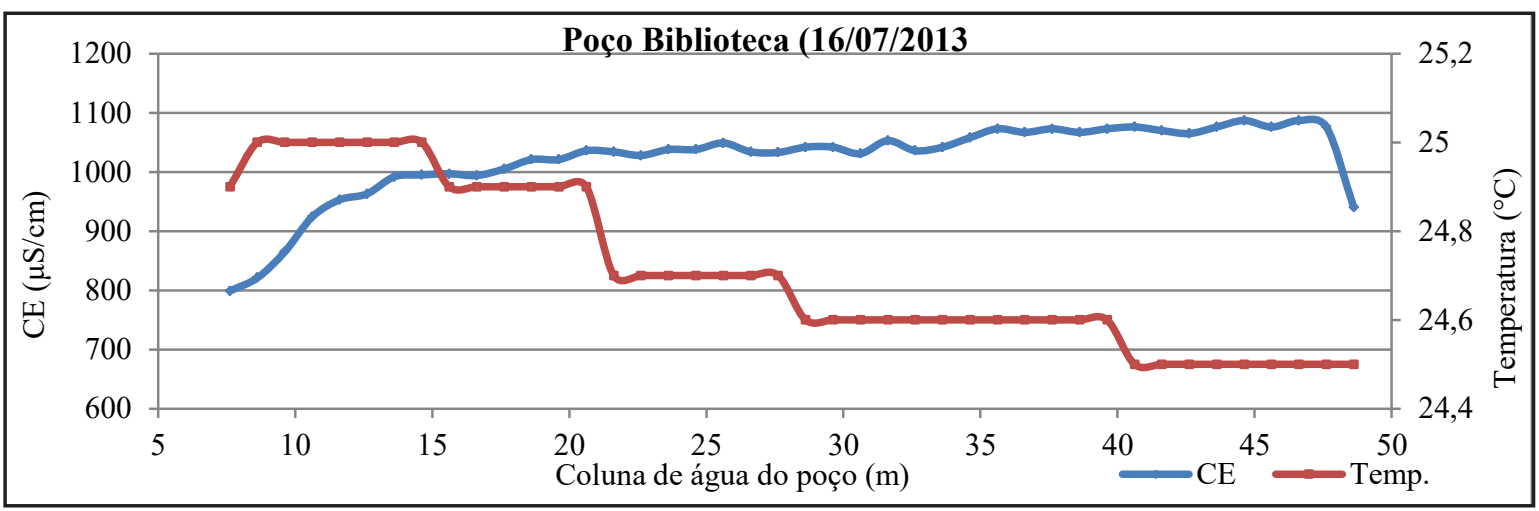

Figura 4 - Variação de CE e temperatura da água ao longo da coluna da água no poço Biblioteca no dia 16/07/2013 Fonte: Trabalho de campo, julho de 2013 


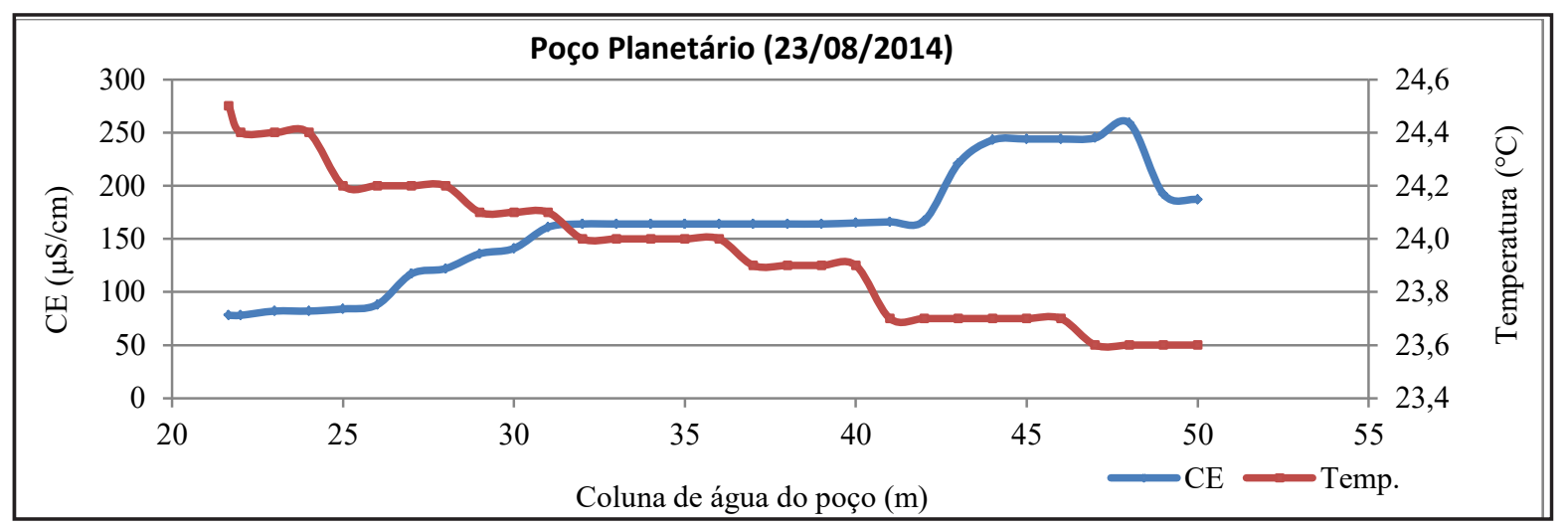

Figura 5 - Variação de CE e temperatura da água ao longo da coluna da água no poço Planetário no dia 23/08/2014. Fonte: Trabalho de campo, agosto de 2014

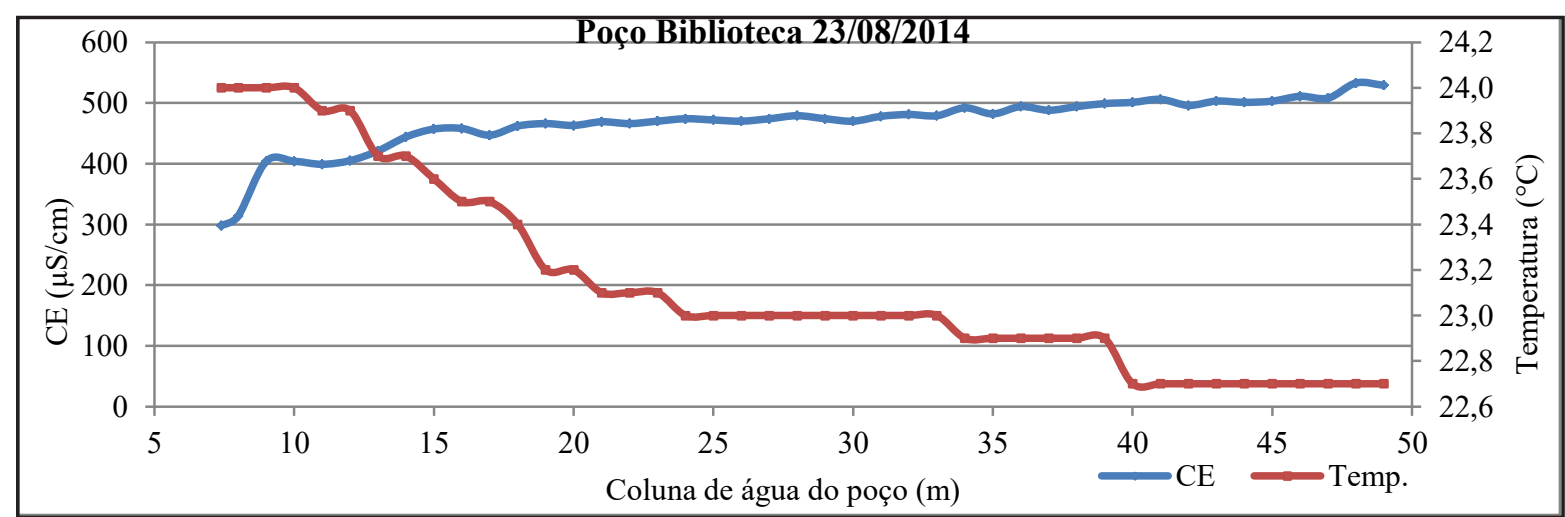

Figura 6 - Variação de CE e temperatura da água ao longo da coluna da água no poço Biblioteca no dia 23/08/2014 Fonte: Trabalho de campo, agosto de 2014

nas duas campanhas de coletas, apresentaram resultados semelhantes quanto ao seu comportamento na coluna da água, sendo que os dados variaram de um máximo de $465 \mu$ S.cm-1 a um mínimo de $181 \mu$ S.cm-1, sendo que esses valores extremos de CE foram encontrados no poço Planetário. Em relação à temperatura os dados tiveram uma amplitude variando de $0,5^{\circ} \mathrm{C}$ a $1,3{ }^{\circ} \mathrm{C}$ e foram registrados no poço Biblioteca, indicando variabilidade (figuras 4 e 6).

A variação de $C E$, geralmente, é dada em uma evolução crescente no perfil da coluna da água, ou seja, à medida que se vai aprofundando o sensor tem-se um aumento no valor da condutividade. Essa característica deve ser melhor estudada em outros períodos do ano, principalmente levando-se em conta o perfil geológico do poço, podendo indicar diferentes entradas de água. Outro fator relevante é quanto os níveis de sais presentes na água, que podem indicar desde perdas de nutrientes do solo até processos de salinização ( $\mathrm{Na}+, \mathrm{Cl}-)$ do solo/ rocha e da água.

As correlações observadas para os dados mostraram serem positivas para a maioria dos dados, sendo que as mais significativas correlações foram entre as temperaturas e os níveis da água em todas as campanhas. Ainda para a CE e os níveis nos poços Biblioteca e Planetário na campanha do dia 23/08/2014, também foi elevada a correlação entre a CE e as temperaturas registradas nessa data.

Nos gráficos das figuras 3, 4, 5 e 6 observa-se o comportamento das variáveis ao longo da coluna da água. Pode-se observar neles, como já foi comentada a tendência da CE em aumentar na coluna da água e a temperatura diminuírem ao longo da coluna em função do aprofundamento no poço.

\section{Conclusões}

Pode-se concluir com o trabalho, que houve uma variação significativa nos parâmetros CE e temperatura na coluna de água nos poços dedicados, sendo que essa variação se dá de forma inversa, crescente para a CE na coluna da água e decrescente para a temperatura.

Houve algumas pequenas variações entre as diferentes datas de coleta para os mesmos poços, contudo constatouse que prevaleceu a uniformidade dos dados na maioria dos casos.

Sugere-se para futuros trabalhos, comparar campanhas em datas nas diferentes estações climáticas do ano, buscando-se avaliar possíveis efeitos sazonais. Ainda sugere-se uma maior cobertura estatística dos dados e estudo do material geológico do perfil do poço captado, complementando com análises químicas para a determinação das concentrações de íons presentes nas águas. 


\section{Agradecimentos}

Agradecimentos aos financiadores à FAPERGS, CAPES, CNPq e FIEX/UFSM.

\section{Referências}

CARMO, J. A. R. DO. (2014) Recarga subterrânea direta e sua influência sobre a temperatura e a condutividade elétrica em microbacias do Rio Vacacaí-Mirim, RS. 2014. 122 f. Dissertação (Mestrado em Engenharia Ambiental) - Universidade Federal de Santa Maria, Santa Maria.

Custodio, E; Silva Junior, G. C. Da. (2008). Conceptos básicos sobre o papel ambiental das águas subterrâneas e os efeitos da sua exploração. Boletín Geológico y Minero v. 119, p. 93-106.

D'Ávila, R. F. (2009). Ensaio metodológico de avaliação de impacto antrópico da Bacia Hidrográfica da UFSM/RS. Santa Maria. 2009. Dissertação de Mestrado. Universidade Federal de Santa Maria.

Drever, J. I. (1997). The geochemistry of natural waters: Surface and grondwater evironments. $3^{\circ}$ ed. Upper Saddle River Prentice Hall, 436p.

LIMA, J. O. G de; FRANÇA, A. M. M.; LOIOLA, H. G. Implicações hidroquímicas da condutividade elétrica e do ín cloreto na qualidade das águas subterrâneas do semiárido cearense. Revista Virtual de Química, v. 6, n. 2, p. 279-292, 2013.

Luiz, T. B. P.; Silvério da Silva, J. L. (2013a). Variabilidade em parâmetros hidrodinâmicos e físico-químicos em poços de monitoramento no Campus da Universidade Federal de Santa Maria, RS. XXVIII Jornada Acadêmica Integrada, 2013. Anais. Santa Maria.

Luiz, T. B. P.; Silvério da Silva, J. L. (2013b). Variabilidade da condutividade elétrica em poços de monitoramento do Campus da UFSM. XX Simpósio Brasileiro De Recursos Hídricos, 2013. Anais. Bento Gonçalves.

Löbler, C. A.; Silverio da Silva, J. L, Bortolotto R. W; Toscani, R. R. (2010). Avaliações dos Níveis dos Poços de Abastecimento do Campus da UFSM. XXV Jornada Acadêmica Integrada, 2010. Anais. Santa Maria.

Löbler, C. A.; Silvério da Silva, J. L.; Toscani, R. R. (20111). Monitoramento dos Poços Tubulares do Campus da UFSM. XXVIJornada Acadêmica Integrada, 2011. Anais. Santa Maria.

Löbler, C. A.; Ertel, T.; Silvério da Silva, J. L. (2012). Relação entre os índices de precipitação e recarga nos poços tubulares do Campus da UFSM. XXVII Jornada Acadêmica Integrada, 2012. Anais. Santa Maria.
Löbler, C. A.; Ertel, T.; Silvério da Silva, J. L. (2013). Estimativas de recarga das águas subterrâneas no Campus da UFSM. XX Simpósio Brasileiro De Recursos Hídricos. Anais, Bento Gonçalves.

Löbler, C. A. (2015). Avaliação quantitativa da recarga das águas subterrâneas na Bacia Escola do Campus da Universidade Federal de Santa Maria. Dissertação de Mestrado (Engenharia Ambiental) Universidade Federal de Santa Maria.

Machado, J. L. F. (2005). Compartimentação espacial e arcabouço hidroestratigráfico do Sistema Aquífero Guarani no Rio Grande do Sul. São Leopoldo. 2005. Tese de Doutorado. Universidade do Vale dos Sinos, São Leopoldo.

Moreira, C. M. D. (2005). Aspectos qualitativos da Água subterrânea no Campus da UFSM. Santa Maria. 2005. Dissertação de Mestrado. Universidade Federal de Santa Maria, Santa Maria.

Marion, F. A; Capoane, V.; Silvério da Silva, J. L. (2007). Avaliação da qualidade da água subterrânea em poço do Campus da UFSM, Santa Maria, RS. Revista Ciência e Natura, v.29, n.1, p. 97-109.

Marion, F. A. (2009). Avaliação da vulnerabilidade das águas subterrâneas por geoprocessamento, no Campus da UFSMRS. Santa Maria. 2009. Dissertação de Mestrado. Universidade Federal de Santa Maria, Santa Maria.

Reckziegel, T. (2012). Modelo Conceitual de Contaminação por Emissão de Efluente no Solo-Bacia Escola CAMPUS/UFSM. Santa Maria. 2012. Dissertação de Mestrado, Universidade Federal de Santa Maria, Santa Maria.

Santiago, M. R. (2010). Análises das Ocorrências Anômalas de Flúor em Águas Subterrâneas. Santa Maria. 2010. Dissertação de Mestrado. Universidade Federal de Santa Maria, Santa Maria.

Schuster, S. L.; Ertel, T.; Löbler, C. A.; Silvério da Silva, J. L. (2013). Comparação de variáveis quantitativas e qualitativas dos poços tubulares Planetários e Biblioteca do Campus da UFSM. XXVIII Jornada Acadêmica Integrada, 2013. Anais. Santa Maria.

Silvério da Silva, J. L., Moreira, C. M. D., Osorio, Q. Da S.; Löbler, C. A. (2012). Captações de água subterrânea no Campus da UFSM, Santa Maria-RS. Revista Monografias Ambientais. v.9, p.1953-1969.

Silvério da Silva, J. L.; Carmo, J. A. R. do. (2013) Monitoramento da condutividade elétrica em poço raso em Zona de Afloramentos do Sistema Aquífero Guarani, RS. In: XX Simposio Brasileiro de Recursos Hidricos, Bento Gonçalves: ABRH. 